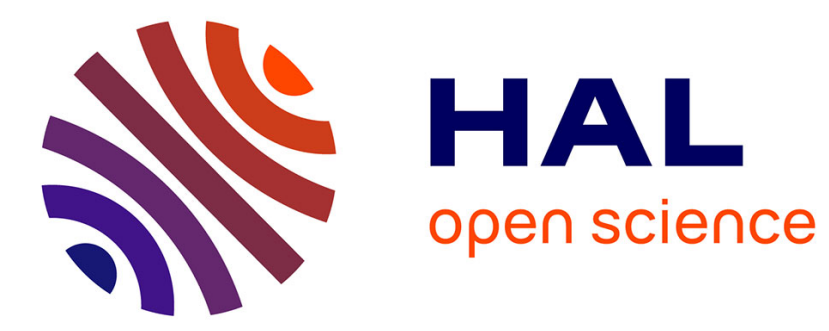

\title{
X-ray reflectivity measurements on Cd-arachidat films
}

\author{
H. Albrecht, Th. Gerber, M. Kinzel, M. Grunze
}

\section{To cite this version:}

H. Albrecht, Th. Gerber, M. Kinzel, M. Grunze. X-ray reflectivity measurements on Cd-arachidat films. Journal de Physique IV Proceedings, 1993, 03 (C8), pp.C8-189-C8-192. 10.1051/jp4:1993838 . jpa-00252269

\section{HAL Id: jpa-00252269 https://hal.science/jpa-00252269}

Submitted on 1 Jan 1993

HAL is a multi-disciplinary open access archive for the deposit and dissemination of scientific research documents, whether they are published or not. The documents may come from teaching and research institutions in France or abroad, or from public or private research centers.
L'archive ouverte pluridisciplinaire HAL, est destinée au dépôt et à la diffusion de documents scientifiques de niveau recherche, publiés ou non, émanant des établissements d'enseignement et de recherche français ou étrangers, des laboratoires publics ou privés. 


\title{
X-ray reflectivity measurements on Cd-arachidat films
}

\author{
H. ALBRECHT, Th. GERBER, M. KINZEL* and M. GRUNZE* \\ Universität Rostock, Fachbereich Physik, Universitätsplatz 3, 18051 Rostock, Germany \\ * Universität Heidelberg, Angewandte Physikalische Chemie am Physikalisch Chemischen Institut, Im \\ Neuenheimer Feld 253, 69120 Heidelberg, Germany
}

\begin{abstract}
LANGMUIR-BLODGETT films of Cd-arachidate with a thickness of 3 and 25 layers were investigated by X-ray reflectivity measurements.

$\mathrm{X}$-ray reflectivity is a non-destructive method for investigations of thin layers or layer systems.

The shape of a reflectivity curve is determined by the complex refraction index $n(z)$ of the substrate and of the layers perpendicular to the surface. In the framework of the dynamical theory, PARRATT [1] gave a recursion formula for the calculation of the interference pattern. The data anaiysis by means of PARRATT's formula is useful for samples with a few layers only, because the number of free parameters increases with the number of layers.

Data analysis using the kinematic theory is possible if we can eliminate the part of total reflection from the pattern. The size of the lattice unit and the electron density can be calculated .

BRAGG peaks up to the fourteenth order are observed. The Patterson function can be calculated by Fourier transform of the corrected intensity function. A ideal intensity function can be constructed with the help of the location and the area of the BRAGG peaks. The Fourier transform of the ideal intensity function gives a ideal Patterson function. The phase problem of $x$-ray diffraction is reduced to the sign of the scattering amplitude for the case of an one dimensional crystal structure. Therefore it is possible to calculate the average electron density of the lattice unit of the layer structure.

The results of the reflectivity measurements on Cd-arachidate films show, that the upmost layer has a higher disorder as the bulk structure.
\end{abstract}

\section{INTRODUCTION}

Thin films of organic compounds are very interesting for several applications in science, for instance to make sensors for organic compounds.

In order to learn something about the layer structure of organic compounds it is necessary to use a well known compound. The results of the examinations are the basis for the exploration of more difficult layer structures. Cd-arachidate is a compound which was examined with several methods. The structure of the $\mathrm{Cd}$-arachidate molecule is well known. The chemical composition of $\mathrm{Cd}$-arachidate is $\mathrm{CH}_{3}-\left[\mathrm{CH}_{2}\right]_{18}$ $\mathrm{COO}_{2} \mathrm{Cd}$. The length of each molecule is about $2.5 \mathrm{~nm}$.

The bonding angle between $\mathrm{CH}_{2}$-groups is $110.5 \mathrm{deg}$, the bonding angle between carbon and hydrogen is $109.6 \mathrm{deg}$.

Cd-arachidate layers are prepared by the LANGMUIR-BLODGETT-method.

Uneven numbers of layers can be deposited on a Si-wafer.

The present paper provides information about X-ray reflectivity measurements of Cd-arachidate films. By means of the dynamical theory a model was calculated for samples consisting of 1 layer or of 3 layers Cdarachidate. We also tried to use the kinematic theory for samples consisting of 3,9 and 25 layers. In this paper the results of PARRATT's formula for 3 layers and the results of the kinematic theory for 25 layers are described. 


\section{X-RAY REFLECTIVITY}

It is well known that the refraction index for X-rays is smaller than one. The refraction index is given by

$$
n=1-\delta-i \cdot \beta
$$

The amount of $\delta$ and $\beta$ are very small, generally in the order of $10^{-5} \ldots 10^{-8}$. $\delta$ and $\beta$ are given by

$$
\begin{aligned}
& N_{a} \ldots \text { number of Avogadro } \quad \lambda \text {...wowelength } \\
& \delta=\frac{N_{a}}{2 \pi} r_{0} \lambda^{2} \sum_{k} \frac{\rho_{k}}{A_{k}} Z_{k}+f_{k}^{\prime} \quad r_{0} \text {....classicalradiusof electron } \quad A_{k} \ldots \text { weight of the } k \text {-thatom } \\
& \beta=\frac{N_{a}}{2 \pi} r_{0} \lambda^{2} \sum_{k} \frac{\rho_{k}}{A_{k}} f_{k}^{\prime \prime}=\frac{\mu \lambda}{4 \pi} \quad \begin{array}{c}
\rho_{k} \ldots \text { density of the } k \text {-thatom } \\
Z_{k} \ldots \text { number of orderof the } k \text {-thatom } \\
f_{k}^{\prime \prime} \text {...imaginarypartof } f
\end{array} \quad \begin{array}{c}
f_{k}^{\prime} \ldots \text { real partof dispersioncorrectionof } f \\
\mu \text {...number of absorption }
\end{array}
\end{aligned}
$$

Because $\mathrm{n}$ is smaller than one total reflection of $\mathrm{X}$-rays exist on the interface air-medium if the angle of incidence is smaller than a critical angle $\Theta_{c}=\sqrt{2 \delta}$.

The atomic structure of a layer system can be found if it is possible to determine $\delta$ and $\beta$. PARRATT gave a recursion formula to calculate these parameters for a symmetrical arrangement of incident beam and detected beam $\left(\Theta-2 \Theta\right.$ arrangement ). The wave vector $\mathbf{k}$ has a component $k_{z}$ only. That means the detected intensity contains information vertical to the surface. The $x$-ray reflectivity curve is given by the solution of the MAXWELL equations for the electric and the magnetic field on every interface between the layers. The PARRATT's formula /1/ describes this solution.

Every layer is characterised by a complex refraction index.

$\delta$ and $\beta$ as well as the thickness of the layer are the free parameters for the calculation of the reflectivity curve by PARRATT. Every layer of $\mathrm{Cd}$-arachidate must be subdivided in 4 boxes (layers) describing the $\mathrm{Cd}$-, $\mathrm{COO}-,\left[\mathrm{CH}_{2}\right]_{18}$-part and the $\mathrm{CH}_{3}$-end of the molecule /3/. The recursion formula of PARRATT is valid for smooth interfaces only. It is necessary to make corrections to described the roughness of the interfaces. The correction of the reflected beam is given by NEVOT and CROCE. For the calculation of the reflectivity curve of 1 layer of $C d$-arachidate we need 15 parameters $(\delta, \beta$ and thickness for every box, roughness for the whole arachidate layer; $\delta, \beta$ and roughness for the substrate). 12 parameters are added for every additional arachidate layer. Figure 1 shows the fit for a sample consisting of 3 layers of $\mathrm{Cd}$-arachidate and the electron density profile in z-direction for the solution.

The evaluation of the samples consisting of 9 and 25 layers is much more difficult because the number of free parameters increases.

That's the reason to find an other way to get information about the layer system.

Cd-arachidate forms a well ordered structure vertical to the wafer surface. This high order causes sharp BRAGG-peaks in the experimental curve. Data analysis using the kinematic theory is possible if we can eliminate the component of the total reflection from of the pattern.

The measured intensity is the Fourier transform of the Patterson function which is the self convolution of the electron density.

$$
\mathrm{I}(\overrightarrow{\mathrm{s}})=\mathrm{F}\left\{\left\langle\rho_{\mathrm{\theta}}(\overrightarrow{\mathrm{r}}) * \rho_{\mathrm{\theta}}(\overrightarrow{\mathrm{r}})\right)\right\}=\mathrm{A}(\overrightarrow{\mathrm{s}}) \cdot \overline{\mathrm{A}(\overrightarrow{\mathrm{s}})}
$$

In the $\Theta-2 \Theta$ arrangement the wave vector has a component in z-direction only. In this case the three dimensional intensity function is reduced to an one dimensional problem. That's why it is possible to get

$$
\text { the amplitude function directly: } \quad I\left(s_{z}\right)=A\left(s_{z}\right)^{2}, \quad A\left(s_{z}\right)=A_{0} \int \rho_{e}(z) \cdot e^{-i \cdot c \cdot s_{x}} d z .
$$

The Fourier transform of the amplitude function is the electron density vertical to the surface.

We used the positions and the areas of the peaks of the experimental curve to build the ideal layer structure vertical to the surface. The ideal structure of the layer system is very well ordered and with 
infinite extension. We find the size of the lattice unit of the one dimensional crystal. Therefore we get information about the tilt angle of the molecules to the surface.

The comparison of the Patterson function of the ideal infinite crystal with the Patterson function of the real system gives information about the disorder in the layer structure.

\section{RESULTS}

Figure 2 shows the experimental curve of the sample consisting of 25 layers of $\mathrm{Cd}$-arachidate. We measured BRAGG-peaks up to the fourteenth order. The corrected intensity function is the quotient of the measured intensity function and the calculated reflectivity on the surface of the whole system $/ 4,5 /$. Figure 3 shows the corrected experimental curve. Drawing the peak order versus the scattering vector yields a straight line (fig. 4) and gives the size of the lattice unit with $(5.52 \pm 0.08) \mathrm{nm}$. That means the tilt angle is zero in the bulk.

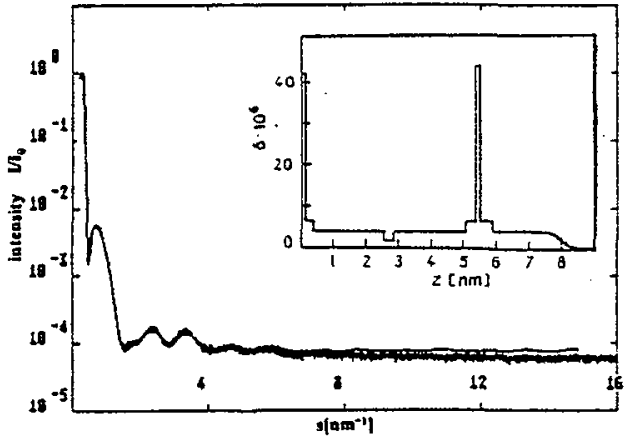

Fig. 1: Exp. curve and model for 3 layers Cdarachidate

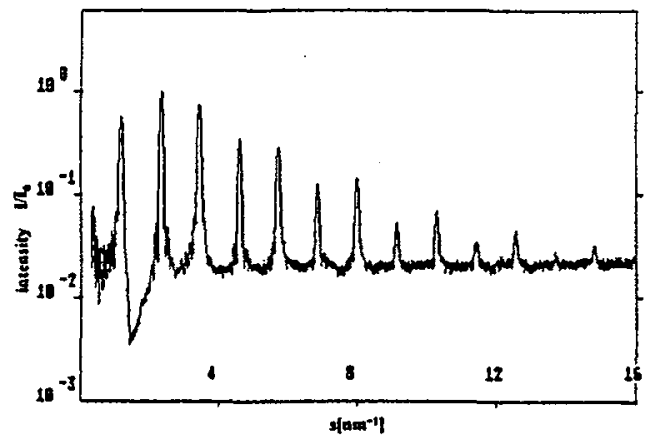

Fig. 3: Corrected exp. curve

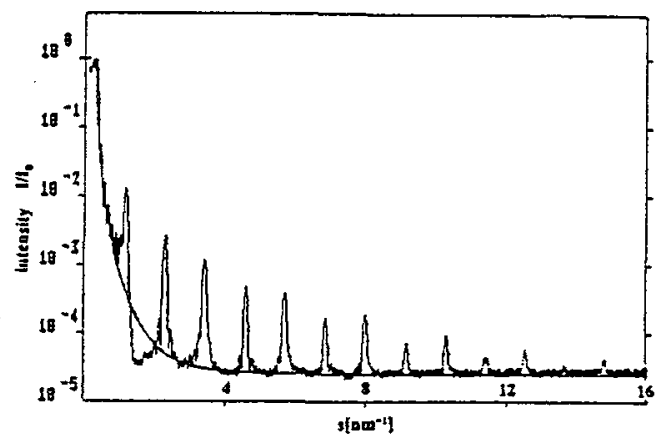

Fig. 2: Exp. curve of 25 layers Cd-arachidate

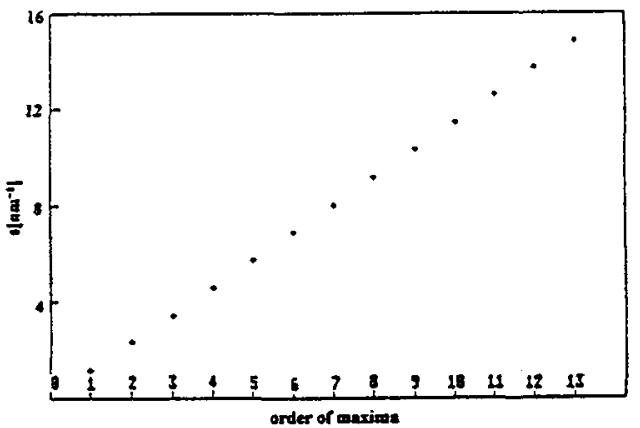

Fig. 4: Peak order versus scattering vector

The Patterson function of the ideal structure (fig. 5) is a periodical function. The large maxima describe the distance between the $\mathrm{Cd}$-atoms. For a real well ordered system of layers of a whole thickness $z$ the Patterson function is the product of the ideal Patterson function and a triangle with the cardinal length of z.

The calculated Patterson function (fig. 6) shows that we haven't a well ordered system. $10 \mathrm{Cd}-\mathrm{Cd}$ distances were found in the function. A system of 25 layers of arachidate is characterised by $12 \mathrm{Cd}-\mathrm{Cd}$ distances. 


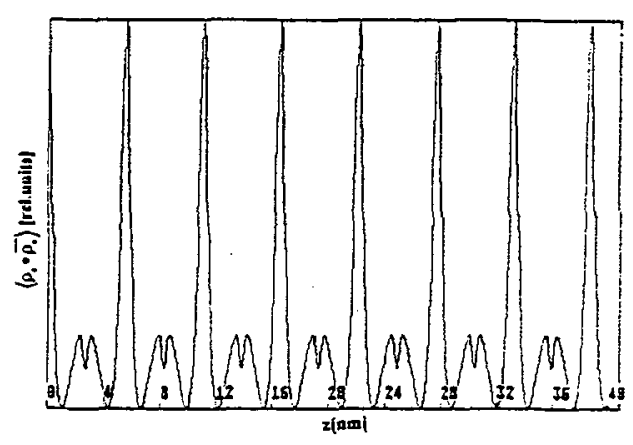

Fig. 5: Patterson function of ideal structure

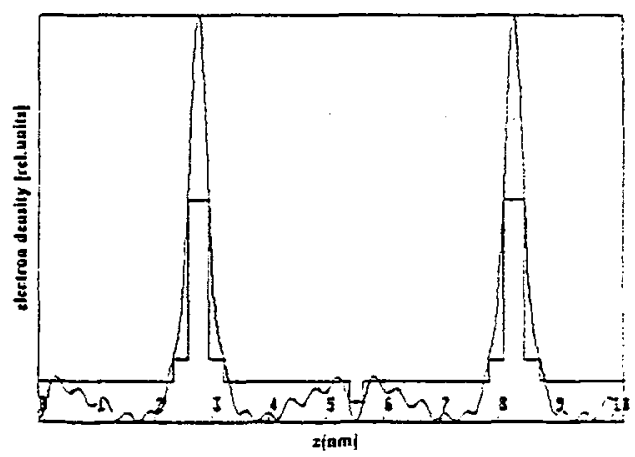

Fig. 7: Electron density profile of the bulk structure

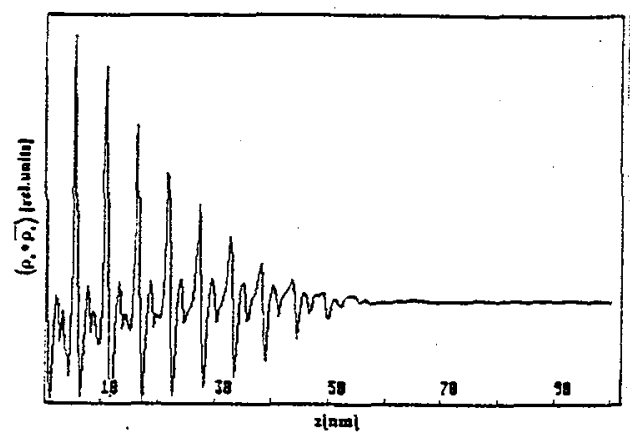

Fig 6: Patterson function of real structure

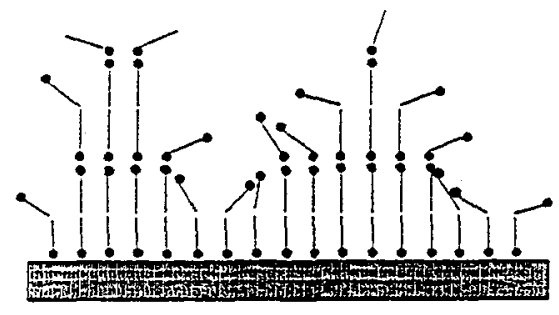

Fig 8: Model of the stnucture of 21 layers Cdarachidate

The deviations is determined of the disordered structure of the layers near the surface. An idea of the structure of the surface of the layer consisting of 25 layers shows fig. 8. At the top of the well ordered bulk structure we found a disordered structure caused by uneven coat.

We get information about the well ordered bulk structure by the Fourier transform of the ideal amplitude function. The square root of the ideal intensity function is the amplitude function that means the Fourier transform is the electron density vertical to the surface (fig. 7).

The information about the well ordered bulk and the disordered surface gives a model like fig. 8 .

\section{ACKNOWLEDGEMENTS}

We gratefully acknowledge the support of this research by the DFG ( grant Ge 667/3-1).

\section{LITERATURE}

[1] PARRATT, L.G.: Phys.Rev. 95 (1954) 359

[2] WARREN, B.E.: X-Ray diffraction; Addison-Wesley (1969)

[3] POMERANTZ, M. ,SEGMÜLLER, A.: Thin Solid Films 18 (1973) 287-294

[4] KJAER, K.:Thin Solid Films, 159 (1988) 17-28

[5] TIDSWELL, I.M.: Phys.Rev. B 41/2 (1990) 